\title{
Neural modelling, control and optimisation of an industrial grinding process
}

\author{
James J. Govindhasamy ${ }^{\mathrm{a}}$, Seán F. McLoone ${ }^{\mathrm{b}}$, George W. Irwin ${ }^{\mathrm{a}, *}$, \\ John J. French ${ }^{\mathrm{c}}$, Richard P. Doyle \\ ${ }^{a}$ Department of Electrical and Electronic Engineering, Intelligent Systems and Control Research Group, Queen's University Belfast, \\ Ashby Building, Stranmillis Road, Belfast BT9 5AH, North Ireland, UK \\ ${ }^{\mathrm{b}}$ Department of Electronic Engineering, National University of Ireland Maynooth, Maynooth, Co. Kildare, Ireland \\ ${ }^{\mathrm{c}}$ Seagate Technology Media Ltd, 99 Dowland Road, Aghanloo Industrial Estate, Limavady BT49 OHR, North Ireland, UK
}

Received 30 September 2003; accepted 4 November 2004

Available online 7 January 2005

\begin{abstract}
This paper describes the development of neural model-based control strategies for the optimisation of an industrial aluminium substrate disk grinding process. The grindstone removal rate varies considerably over a stone life and is a highly nonlinear function of process variables. Using historical grindstone performance data, a NARX-based neural network model is developed. This model is then used to implement a direct inverse controller and an internal model controller based on the process settings and previous removal rates. Preliminary plant investigations show that thickness defects can be reduced by $50 \%$ or more, compared to other schemes employed.
\end{abstract}

(C) 2004 Elsevier Ltd. All rights reserved.

Keywords: Neural networks; Nonlinear modelling; NARX models; Disk grinding process; Multilayer perceptrons; Direct inverse model control; Internal model control

\section{Introduction}

Artificial neural networks (ANNs) have been successfully used in many process control applications. Their ability to approximate arbitrary nonlinear vector functions, combined with dynamic elements has yielded a powerful tool for modelling nonlinear dynamical systems. A recent survey by Dote and Ovaska (2001) reviewing a decade of industrial innovations employing soft computing techniques, identified ANNs as having being successfully applied across a broad spectrum of sectors, including manufacturing automation. They estimate that, on the whole, publications on applications of soft computing techniques have increased steadily in

\footnotetext{
*Corresponding author. Tel.: + 442890335439 ; fax: +442890664265 .

E-mail address: g.irwin@ee.qub.ac.uk (G.W. Irwin).
}

the past 10 years, with manufacturing automation and robotics leading the growth by an average of $1.06 \%$ a year.

Other reviews on applications of ANNs in manufacturing and industrial processes include Warwick, Irwin, and Hunt (1992), Irwin, O'Reilly, Lightbody, Brown, and Swidenbank (1995a), Irwin, Warwick, and Hunt (1995b), Hunt, Irwin, and Warwick (1995) who report on applications of neural networks in control and system optimisation, Hussain (1999) who surveys the chemical process industries and Udo (1992) and Zhang and Huang (1995) who review applications such as resource allocation, scheduling, injection moulding, milling, metal cutting, arc welding and spray painting.

Furthermore, a recent European study on the Stimulation Initiative for European Neural Applications, Esprit Project 9811 (SIENA project) indicates ANNs are used in $39 \%$ of the production or 
manufacturing sectors, with $35 \%$ of the usage related to control, monitoring, modelling and optimisation functions (Heikkonen \& Lampinen, 1999). The potential of ANNs has also been identified in the United Kingdom (UK) with the Department and Trade and Industry initiating a $£ 5.7 \mathrm{M}$ awareness campaign on the applicability of ANNs in industry (Lennox, Montague, Frith, Gent, \& Bevan, 2001).

Machining processes alone account for a significant portion of manufacturing operations (Shin, Chen, \& Kumara, 1992). It is estimated that $15 \%$ of the value of all mechanical components manufactured worldwide is derived from machining operations (Merchant, 1998). With neural network properties in mind, it is beneficial to apply them to these highly nonlinear industrial applications, where reliable and effective models are needed for operational planning and control.

This paper presents the results of collaborative research with Seagate Technology Media (Ireland) Ltd (Seagate Technology: Company Facts Sheet, 2002) on the optimisation of a ring grinding process. Seagate is the world's largest manufacturer of disc drives, with 15 million units shipped in the first quarter of 2002. The main component of the disk drive, the aluminium substrate disc, is manufactured at their plant in Northern Ireland. The subject of this study is the optimisation of a grinding process used to machine the aluminium substrate disks to a desired thickness. The process involves 12 parts being simultaneously ground at each machine cycle. A proprietary thickness control algorithm, which employs thickness measurements before and after grinding, is used to calculate the average stock removal rate for each machine cycle. This approach does not adequately account for the nonlinear variation of the cycle-to-cycle removal rate, hence the call for a better control methodology.

A number of researchers have investigated the application of ANNs for grinding process optimisation. Chryssoloursis, Guillot, and Domroese (1988) in their work on a sequential decision-making method used three modelling techniques - multiple regression, group method of data handling and ANNs on orthogonal machining of aluminium tubes. They found that ANNs provided the best overall accuracy in estimating the state variables of the process.

Shin et al. (1992) developed an intelligent grinding advisory system based on fuzzy logic inference for assisting process engineers to design new grinding processes. Liao and Chen (1994) used experimental data to model a creep feed grinding process using simple backpropagation for training the networks. Shin and Vishnupad (1996) modelled a grinding system using ANNs based on data generated by an analytical model of the grinding operation. However, the control was performed by a fuzzy logic controller.
Brinkmeier, Tšnshoff, Czenkusch, and Heinzel (1998) described different methods for modelling and optimisation of a grinding process where the quality properties of the process like surface texture and finishing are required to be measured. The process was then modelled using ANNs, fuzzy sets, standard multiple regression methods and deterministic methods which were finally optimised using genetic algorithms (GAs). Chen and Kumara (1998) developed an advisory system to optimise surface grinding processes using fuzzy and neural networks. They first built a fuzzy optimiser, and used the trajectory it generated to train the corresponding neural optimiser.

Finally, Lee and Shin (2001), developed a static model of a ring grinding process similar to that under consideration in the current study, which they adapted on-line, and used it as the basis for a direct adaptive inverse controller. They reported excellent simulation results confirming the applicability of ANNs to grinding process optimisation.

The grinding process modelling research outlined above is based on the use of static models and no attempt has been made to date to account for any underlying process dynamics. The main contribution of this paper is that the dynamic process variations are captured using past values of the process as inputs to a Nonlinear AutoRegressive with eXogenous input (NARX) model. A novel methodology of separately modelling the high- and lowfrequency variations in the process data is also proposed. The development and testing of this NARX model is described in detail. Test results on actual grindstone data indicate that the resultant neural network grindstone model is vastly superior to the static scheme proposed by Lee and Shin (2001).

Utilising the neural NARX model as the basis for controller design several alternative control schemes are investigated and shown to yield much tighter process control than the existing proprietary control scheme and the direct inverse scheme proposed by Lee and Shin (2002).

The remainder of the paper is organised as follows: Section 2 describes the grinding process application, while in Section 3, the relevant process variables are identified along with preliminary model development. Section 4 introduces the generalised MLP and develops the new NARX-based grinding process model proposed. Then in Section 5, various control schemes are described and their performance on actual grindstone data is reported in Section 6. Finally, the conclusions of the study and suggestions for further work are given in Section 7.

\section{Ring grinding process}

The aluminium substrate disks are ground in batches of 12 between two grindstones, as shown in Figs. 1 


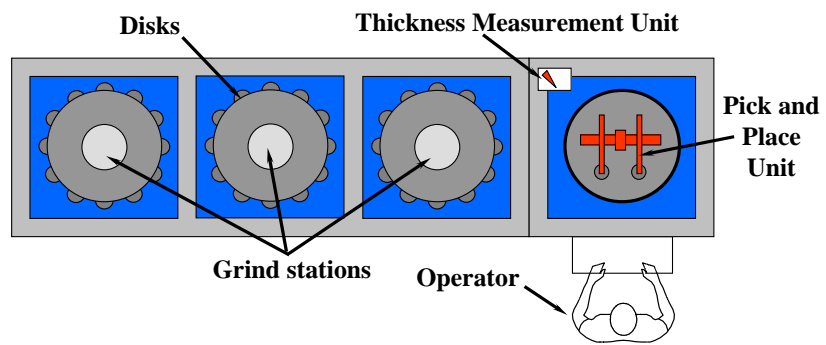

Fig. 1. Layout of the ring grinding process.

and 2. The stones can be moved apart to allow loading and unloading of the disks using a pick and place unit. During operation the grindstones are rotated in opposite directions with pressure applied to the upper stone. This causes the substrate disks between them to rotate ensuring uniform grinding of their surfaces. The rate at which the disks are ground, called the removal rate, is the critical variable. It varies depending on a number of parameters including stone wear, exerted pressure, lubricant viscosity and coolant flow rate. The initial thickness of the disks also varies, although the disks in any one batch are sorted to be approximately the same thickness (i.e. within $\pm 1.25 \mu \mathrm{m}$ ). Disk thickness is measured in situ using a non-contact thickness gauge known as an air gauge ${ }^{1}$. Currently, the thickness of one disk from each batch is measured before the batch is ground. The system controller determines the actual removal rate from the previous batch and estimates the current value of removal rate using a proprietary control law. It predicts how much material has to be removed by subtracting the target thickness from the input thickness and then calculates the necessary grinding duration for the current batch.

When the grinding is completed, the selected disk is measured again. If it is within specification, then the whole batch is passed. If the disk is too thick (above the upper specification limit), the disks are ground again (i.e. reworked) but if the disk is too thin (below the lower specification limit), the batch is rejected. When a grindstone is newly installed (i.e. replaced due to wear), the pressure is initially set to a low value and then gradually increased to an upper limit to counteract the stone deterioration, which in turn increases the removal rate. Subsequently, the removal rate decreases until a stage is reached where it is so low that the grindstone has to be resurfaced. This is done by slicing off the worn part of the grindstone. Once re-installed the whole process is repeated.

\footnotetext{
${ }^{1}$ This uses the relationship between the pressure of a jet of air from the gauge nozzle and the gap between the nozzle and the aluminium substrate, to determine the gap width and hence disk thickness.
}

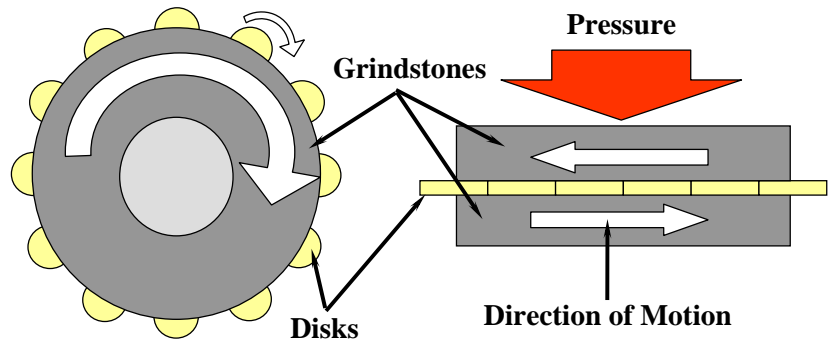

Fig. 2. The ring grinding process.

\section{Preliminary modelling work}

The main objective of this investigation is to develop an accurate control scheme for the thickness of the aluminium substrate disks in order to minimise the number of out of specification disks produced by the grinding process. This process optimisation will be achieved through manipulation of the grind cycle time. As almost all advanced control methods are model based, the first step is to identify an accurate process model. The model developed here is one which predicts the grindstone removal rate for each grind cycle on the basis of the current state of the process. Since analytical modelling is not feasible, data-based modelling techniques have to be employed. This will now be discussed.

Various process variables are logged for each grind cycle as part of the company's own process performance-monitoring procedure. These include the current removal rate, the pressure between the grindstones, the cumulative cycle time and the idle time. Cumulative cycle time is logged as it is an indication of wear and aging of the grindstones while idle time is monitored because of its effect on grindstone operating temperature, which in turn impacts on the removal rate. A summary of these variables and the identifiers used for them in this paper is provided in Table 1 .

Lifetime data for 5 different grindstones (numbered 1-5) were available for this research.

The data was detrended and normalised to lie within the interval $[-1 ; 1]$. The removal rate data contained high-frequency variations, which made it difficult to model with low-order models. Furthermore, these highfrequency variations were unaffected by $p_{k}$ or $c c t_{k}$ as these variables are of low bandwidth. Consequently, the data was divided into its low- and high-frequency components with each one modelled separately and then combined to produce the final prediction of the removal rate as illustrated in Fig. 3.

This partioning was achieved by filtering using a 10point moving average (MA) filter (Masters, 1995). The filter order was deduced by performing correlation tests on the residuals to ensure that they contained no significant information. Fig. 4 provides a snapshot of the actual removal rate data before and after filtering. 
Table 1

Grinding process variables

\begin{tabular}{ll}
\hline Variables & Definition \\
\hline Removal rate, $r_{k}$ & Rate of material removal from a disk during the grind cycle \\
Previous removal rate, $r_{k-1}$ & Removal rate from the previous grind cycle \\
Cycle time, $c_{k}$ & Cycle time of the current grind cycle \\
Cumulative cycle time, $c c t_{k}$ & Sum of all previous cycle times since the grindstone was last resurfaced \\
Pressure, $p_{k}$ & Pressure between the grindstones \\
Idle time, $i t_{k}$ & Length of time that the grindstone remains idle before the next batch of disks are ground \\
Loading thickness, $t_{k}^{L}$ & Thickness of the disk before the grinding process begins \\
Unloading thickness, $t_{k}$ & Thickness of the disk after the completion of the grinding process \\
Target thickness, $t_{k}^{S P}$ & Desired thickness required for each grind cycle \\
Upper control limit, $u c l_{k}$ & Upper control thickness limit specification \\
Lower control limit, $l c l_{k}$ & Lower control thickness limit specification \\
\hline
\end{tabular}

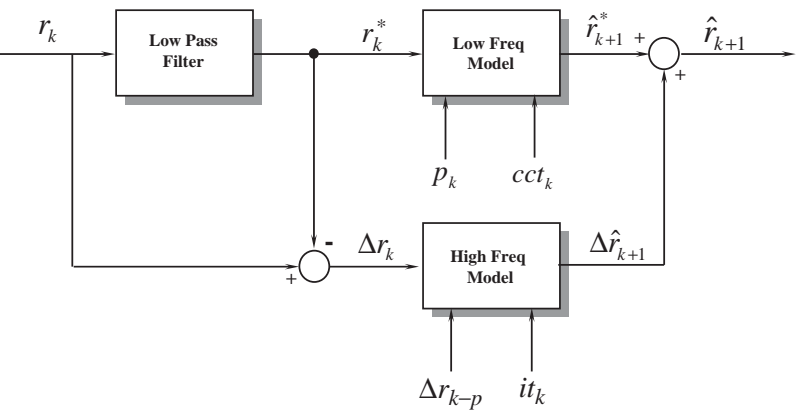

Fig. 3. Removal rate prediction model.

Fig. 5 shows the variations in all the variables used in the process modelling for grindstone 1 . The highfrequency data, $\Delta r_{k}$, plotted in Fig. 6, is simply the residual after the filtered data has been subtracted from the raw measurement of removal rate data.

This partitioning of the modelling problem allows low-order models to be obtained. Initial attempts at modelling the unfiltered data concluded that models of order 10 were required to obtain acceptable performance. In effect the additional terms inherently provide the filtering needed. However, this is an unsatisfactory approach as the parameter space is much larger.

The existing proprietary controller will be used as a base reference for evaluating the performance of the models developed in the following sections. Since this controller is essentially model-free, it does not generate an explicit removal rate prediction, $\hat{r}_{k}$. However, this can be inferred from the generated cycle time, $c_{k}$, using the formula

$\hat{r}_{k}=\frac{t_{k}^{L}-t_{k}^{S P}}{c_{k}}$.

Here, $t_{k}^{L}$ is the loading thickness and $t_{k}^{S P}$ is the setpoint or target thickness. Note that the actual removal rate, $r_{k}$, is obtained by replacing $t_{k}^{S P}$ by the measured

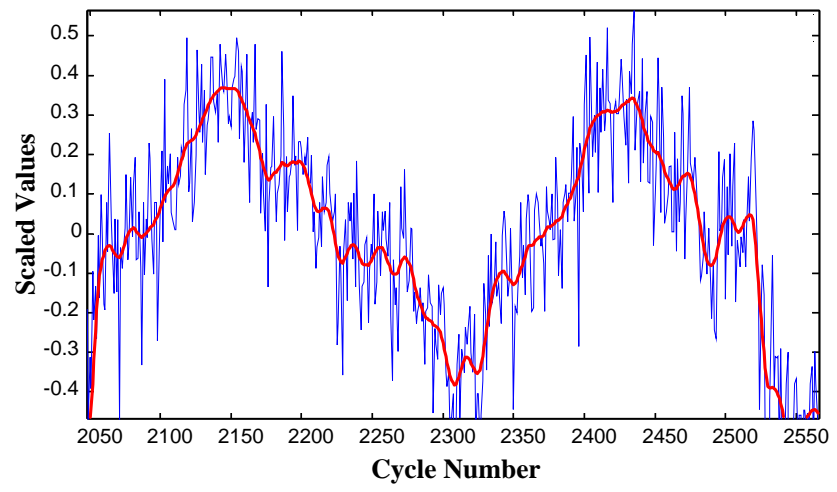

Fig. 4. The removal rate, $r_{k}$, after being filtered and conditioned (-Raw data, -Filtered data).

unloading thickness, $t_{k}$, in the above formula, that is

$r_{k}=\frac{t_{k}^{L}-t_{k}}{c_{k}}$.

Fig. 7 compares the predicted filtered removal rate, $\hat{r}_{k}$, and the actual filtered removal rate, $r_{k}$, for grindstone 1 . The accuracy of the prediction is measured in terms of the percentage normalised mean prediction error (MPE), defined as

$M P E=\frac{1}{n} \sum_{k=1}^{n} \frac{\left|r_{k}-\hat{r}_{k}\right|}{\sigma_{r}} \times 100$.

In this case the MPE for grindstone 1 is $6.8 \% .^{2}$ This is considered a reasonable performance and is consistent with the low number of rejects observed on the process with the existing controller. The occurrence of rejects can be explained by the observation that locally the MPE performance can be much poorer as demonstrated

\footnotetext{
${ }^{2}$ The variance is defined as $v_{r}=\operatorname{var}\left(r_{k}\right)=E\left[\left(r_{k}-\mu_{r}\right)^{2}\right]$, where $\mu_{r}=$ $E\left(r_{k}\right)$ is the mean and $\left.\sigma_{r}=\operatorname{std}\left(r_{k}\right)=\sqrt{[}\right] v_{r}$ is the standard deviation of variable $r_{k}$, respectively, and $n$ is the number of data samples.
} 
by the highlighted data segment in Fig. 7. Consequently, when assessing model performance, both local and global MPE need to be considered.

An investigation into linear modelling (Fig. 8) concluded that the best model for the filtered portion

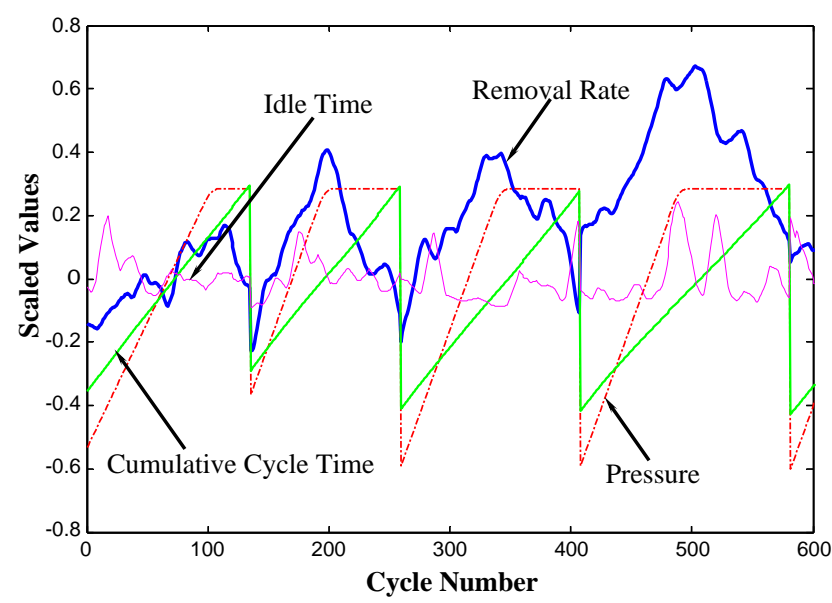

Fig. 5. The variables used in the modelling of the grinding process.

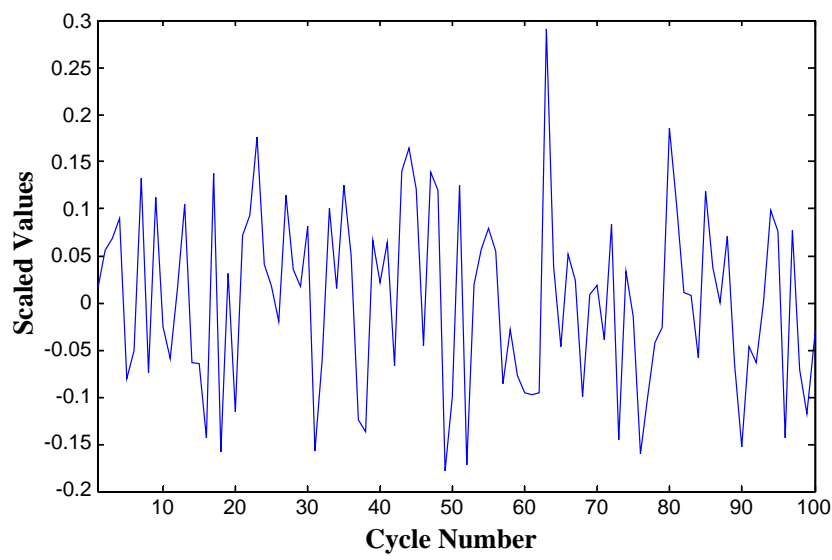

Fig. 6. High-frequency data sample, $\Delta r_{k}$ (the residual after the filtered data is subtracted from the raw data). was of the form:

$$
\begin{aligned}
\hat{r}_{k}^{*}= & a_{1} \hat{r}_{k-1}+a_{2} \hat{r}_{k-3}+a_{3} \hat{r}_{k-5}+b_{1} p_{k} \\
& +b_{2} c c t_{k}+b_{3} c c t_{k-1} .
\end{aligned}
$$

Modelling accuracy was subsequently further improved by an average of $3.8 \%$ (see Section 4.3 ) when the filtered model was augmented with a high-frequency variation model:

$\Delta \hat{r}_{k}=c_{1} \Delta r_{k-1}+c_{2} \Delta r_{k-3}+c_{3} \Delta r_{k-5}+d_{1} i t_{k}$.

However, the overall result was still quite poor. It is evident that while the data does indeed contain linear trends, there are also significant nonlinear trends. The obvious next step was to form a linear model and to augment it with nonlinear terms, as depicted in Fig. 9. The generalised multilayer perceptron (GMLP) provides an ideal framework for doing this, as described in the next section.

\section{Generalised multilayer perceptron (GMLP) for process modelling and control}

The generalised MLP (Fig. 10) is an extension of the standard one. The addition of direct connections, from the inputs to the outputs introduces weights between nodes in non-adjacent layers. The GMLP provides an ideal tool for modelling both the linear and the nonlinear dynamic components of the plant as it can be initialised as a linear model and then adapted to produce the required nonlinear model (Sjöberg, 1997). GMLPs with multiple hidden layers are not used here as, given enough hidden neurons, a single-layer GMLP is sufficient to approximate any continuous function to an arbitrary degree of accuracy. This follows directly from the universal approximation results for MLPs (Hornik, 1989; Cybenko, 1989) as MLPs are a subset of the functions represented by GMLPs.

Here GMLPs with sigmoidal nonlinear activation functions, $a($.$) , in the hidden layer and linear activation$ functions $(y=x)$ in the output layer were employed.

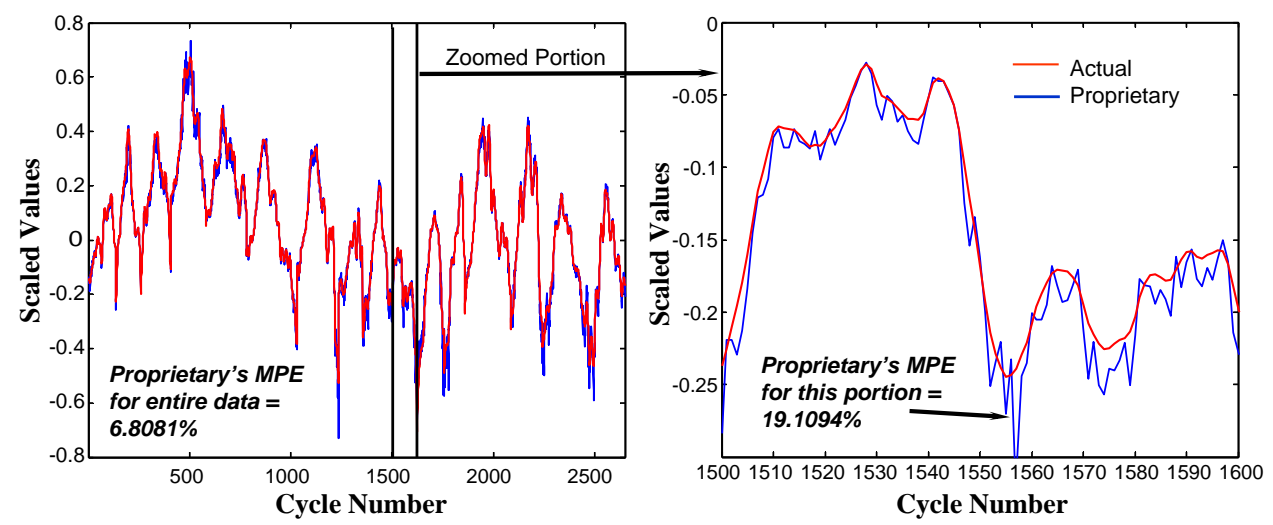

Fig. 7. Example of the filtered removal rate, $r_{k}^{*}$, prediction from the proprietary scheme. 


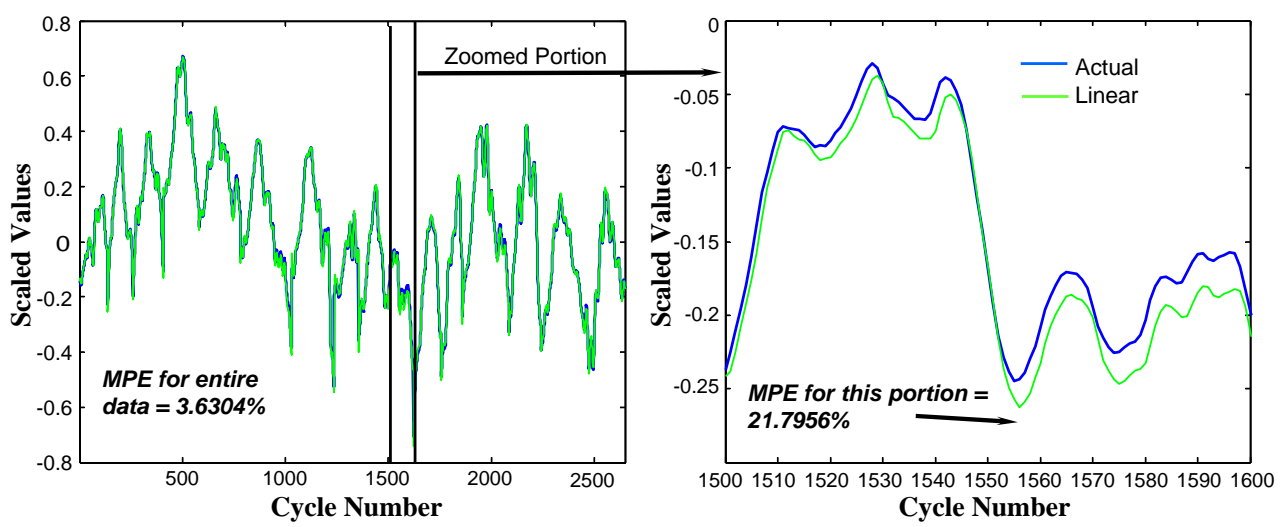

Fig. 8. Sample of the linear model prediction of the filtered removal rate, $r_{k}^{*}$.

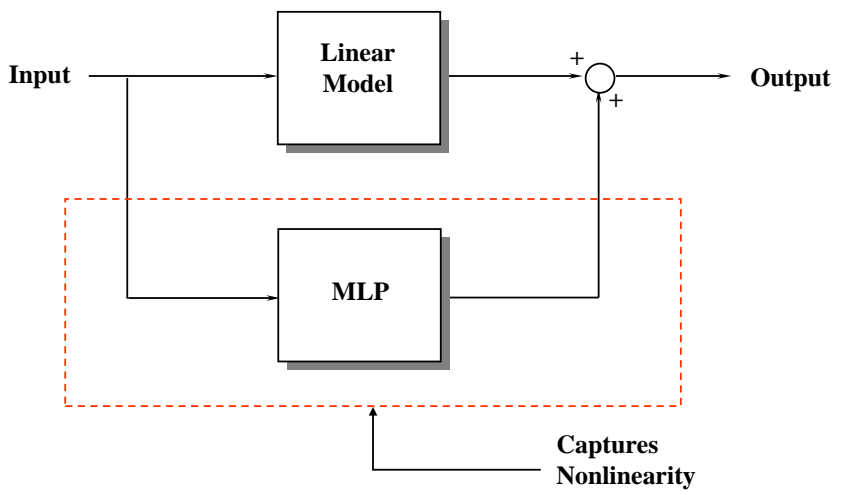

Fig. 9. Block diagram of the nonlinear modelling approach.

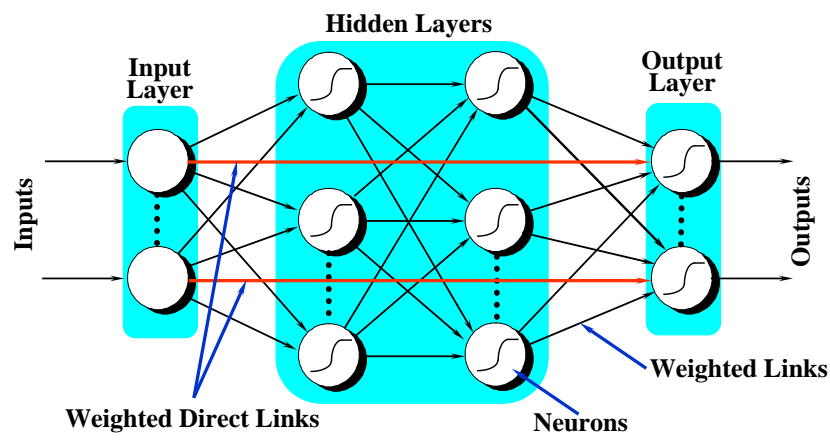

Fig. 10. The generalised multilayer preceptron (GMLP) architecture.

The overall input-output mapping for a multi-input, single-output, single hidden layer GMLP with a linear output neuron can be expressed mathematically as

$$
\begin{aligned}
y & =g(x, w) \\
& =\sum_{j=1}^{N_{h}} w_{l}^{L} a\left(\sum_{i=1}^{N_{i}} w_{l j}^{N L} x_{i}+b_{j}\right)+\sum_{i=1}^{N_{i}} w_{l}^{D L} x_{i}+d,
\end{aligned}
$$

where $N_{i}$ and $N_{h}$ are the number of inputs and hidden layer neurons, respectively, $y$, is the network output and $x_{i}$ is the $i$ th element of the network input vector $\boldsymbol{x}$. The various weights which make up the overall weight vector $\boldsymbol{w}$ are: $w_{j}^{L}$ is the weight between the $j$ th neuron in the hidden layer and the linear $(L)$ output neuron; $w_{i j}^{N L}$ is the weight between the $i$ th input and the $j$ th nonlinear hidden $(N L)$ layer neuron; $w_{i}^{D L}$ is the weight between the $i$ th input and the linear $(L)$ output neuron; $b_{i}$ is the bias on the $j$ th hidden neuron and $d$ is the bias on the linear output neuron.

In this work, the Full Memory Broyden-Fletcher-Shanno-Goldfarb (BFGS) algorithm, a quasi-Newton method was used for training (McLoone \& Irwin, 1997).

For NARX modelling, the filtered removal rate was estimated as a function of previous filtered removal rates, $r_{k-1}^{*}, r_{k-3}^{*}$ and $r_{k-5}^{*}$, current pressure, $p_{k}$, and the current and past cumulative cycle times, $c c t_{k}$ and $c c t_{k-1}$. Thus,

$\hat{r}_{k}^{*}=f\left(\hat{r}_{k-1}, \hat{r}_{k-3}, \hat{r}_{k-5}, p_{k}, c c t_{k}, c c t_{k-1}\right)$,

where the GMLP is trained to learn the unknown mapping of $f($.$) . Similarly, the high-frequency removal$ rate variation, $\Delta \hat{r}_{k}$, was estimated as a function of previous values, $\Delta r_{k-1}, \Delta r_{k-3}$ and $\Delta r_{k-5}$, and current idle time, that is

$\Delta \hat{r}_{k}=g\left(\Delta r_{k-1}, \Delta r_{k-3}, \Delta r_{k-5}, i t_{k}\right)$.

Note that the selection of the filtered and highfrequency removal rate variations interval were chosen to avoid ill-conditioning in the prediction. The resulting neural model is illustrated schematically in Fig. 11.

In the remainder of the paper, the single block diagram on the right and the acronym $D N N M$ will be used to represent this model.

The performance index used in training was the meansquared-error (MSE), defined as

$M S E=\frac{1}{n} \sum_{i=1}^{n}\left(r_{i}-\hat{r}_{i}\right)^{2}$, 


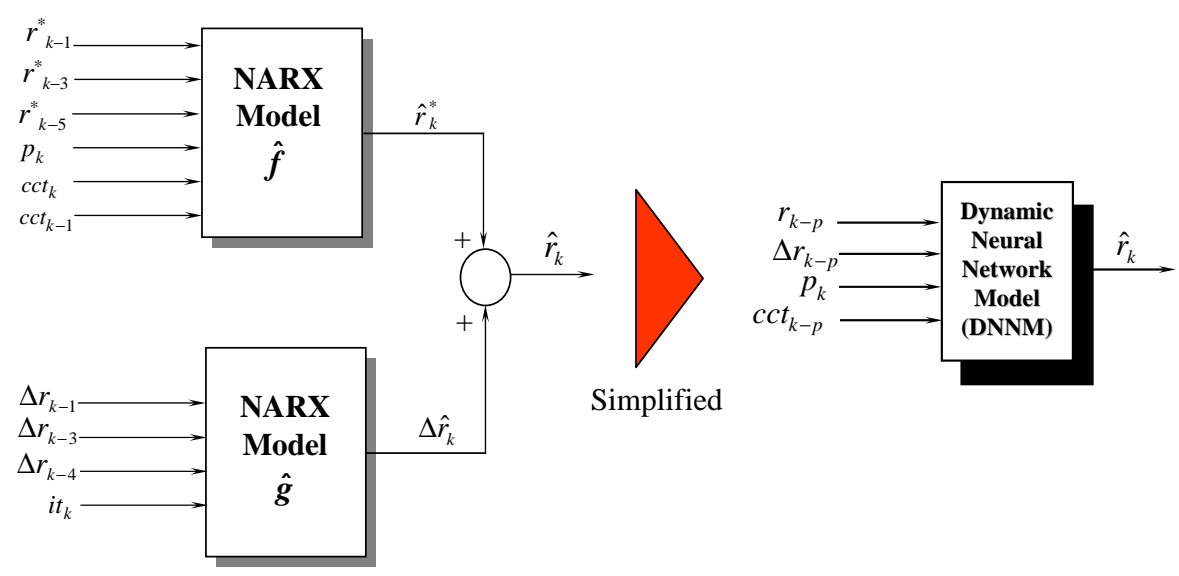

Fig. 11. Schematic of proposed neural network grinding model.
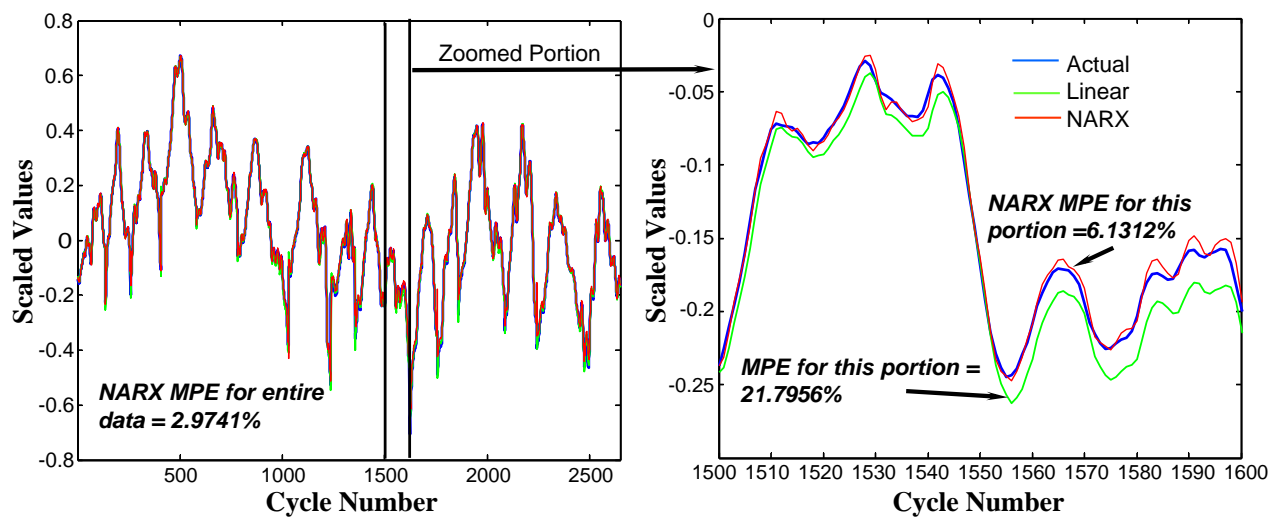

Fig. 12. Comparison of the prediction and actual filtered removal rate for grindstone 1.

where $r_{i}$ is the actual removal rate while $\hat{r}_{i}$ is the estimate and $n$ is the number of data samples. The GMLP networks were trained using the first 600 grindstone data points, with the remainder of the data retained for validation. The goal was to minimise the MSE on this validation data set. This procedure was employed to obtain low- and high-frequency removal rate models for each of the 5 grindstones. After experimenting with different architectures, the network that was found to produce the minimum MSE error was a (6-5-1) one for the filtered data and a (4-5-1) network for the highvariation data. The networks were initialised 10 times where the weights are initialised randomly each time, and the best results recorded for each stone. All analysis was performed offline using Matlab ${ }^{\text {TM }}$. Fig. 12 compares the filtered data GMLP model obtained for grindstone 1 with the corresponding linear model and clearly shows the benefit of moving to a nonlinear modelling framework. Globally, the MPE has been reduced by $18 \%$ compared to the earlier linear model, while locally the reduction is of the order of $71 \%$.

\subsection{Predict-correct scheme for improved removal rate prediction}

Error feedback can also be used to compensate for low-frequency offsets in the ANN removal rate model prediction. This "predict-correct" (PC) technique uses past plant outputs and the corresponding model predictions to generate a correction to the current estimate $\hat{r}_{k}^{*}$ and successful applications have been reported in Rovlak and Corlis (1991), Willis, Di Massimo, Montague, Tham, and Morris (1991), Lightbody (1993), and Irwin et al. (1995a, b). The PC scheme is usually implemented as follows:

$\hat{r}_{k}^{*}=\hat{r}_{k}+\frac{1}{N} \sum_{j=1}^{N}\left(r_{k-j}-\hat{r}_{k-j}\right)$.

A first-order PC was incorporated into the DNNM removal rate predictor as shown in Fig. 13. The performance of the PC scheme for grindstone 1 is 
illustrated in Fig. 14. A modest improvement in both the global and local MPE is observed.

\subsection{Lee and Shin static model}

In contrast to our dynamic modelling approach, Lee and Shin (2001) used a static model for predicting removal rate. This was based on a radial basis function $(\mathrm{RBF})$ neural network, with cumulative material removed, $\sum \Delta t_{k}$ and current pressure, $p_{k}$ as inputs and removal rate, $r_{k}$, as the output as shown in Fig. 15.

A study of this model showed that it was not able to perform adequately for this grinding process. Fig. 16 shows the modelling performance obtained on stone 1 where the static model clearly cannot capture the dynamic variation in the process.

\subsection{Modelling the high-frequency removal rate variation}

The previous sub-sections have confirmed that accurate modelling of the low-frequency removal rate variation is possible using the NARX approach. Modelling of the high-frequency component, however, proved to be much less successful. While this component accounted for between $11 \%$ and $26 \%$ of the total

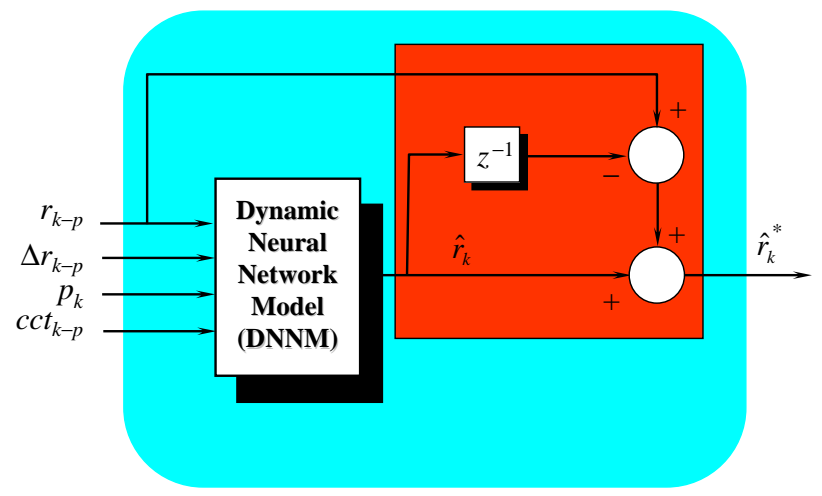

Fig. 13. The predict correct (PC) scheme. removal rate variation in each grindstone, modelling was only able to explain somewhere between $1.5 \%$ and $9 \%$ of this variation. A typical result for grindstone 1 is shown in Fig. 17.

Table 2 provides a summary of the performance of linear and NARX modelling of the high-frequency (H.F.) removal rate data for each grindstone and also provides some statistics on this data. These include the variance of the actual removal rate, the variance represented by the high-frequency data, and the percentage of the high-frequency variation explained by the linear and NARX models. As can be seen, while NARX models are superior to linear models at predicting the high-frequency variation, overall the predictability of the data is very low.

The residuals of the high-frequency data models were found to be normally distributed and uncorrelated as illustrated in Fig. 18 for grindstone 1, leading to the conclusion that the remaining unmodelled removal rate variation is Gaussian white noise. This can be attributed to measurement noise generated by the thickness gauge used to measure the loading and unloading thicknesses utilised in the calculation of removal rate (see Eq. (3.2)). This is supported by the observation that the estimated noise variance (variance of the residual shown in Table 2) is consistent across all grindstones.

\section{Grinding process control}

Having successfully identified a model of the removal rate, the next step is to design an appropriate thickness control system. In this section, a number of different

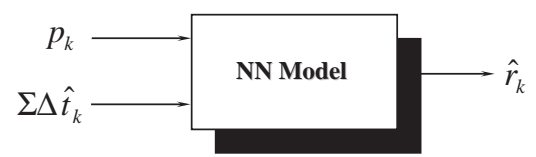

Fig. 15. Static removal rate model proposed by Lee and Shin (2001).

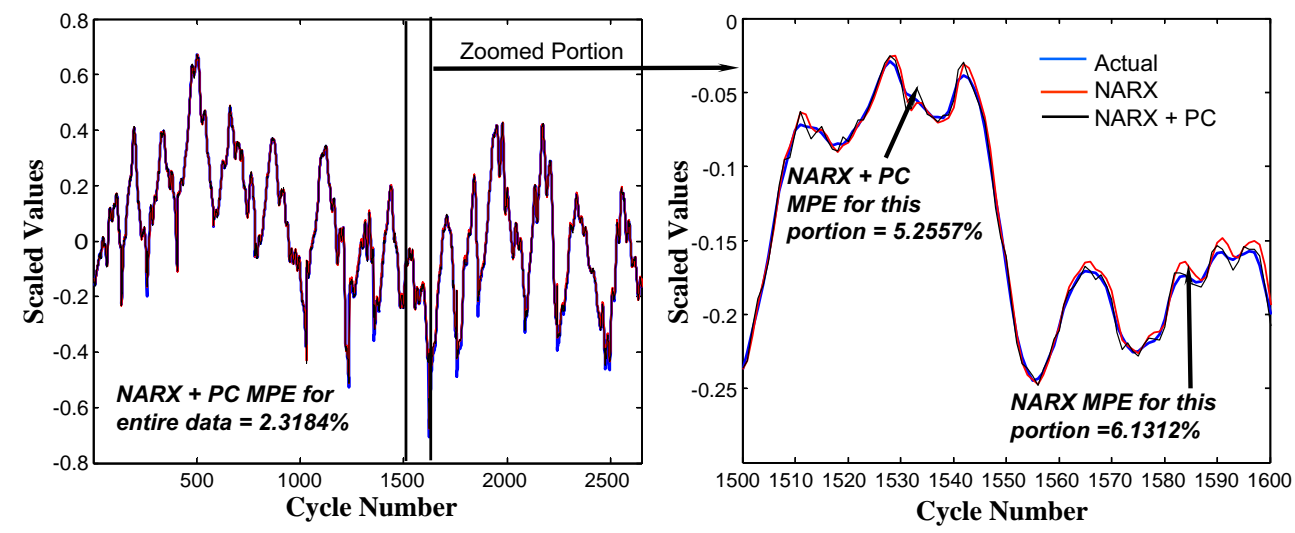

Fig. 14. Comparison of the filtered removal rate predictions from the NARX model, the NARX model with predict correct (PC) and the actual for grindstone 1 . 


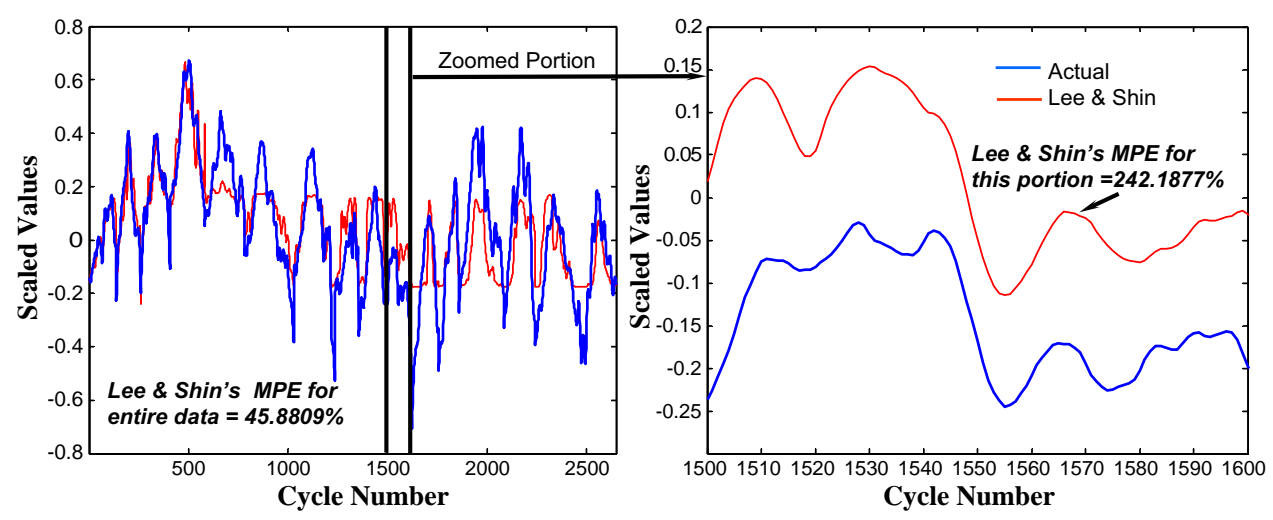

Fig. 16. Comparison of the filtered removal rate predictions from Lee and Shin's static model and the actual for grindstone 1.

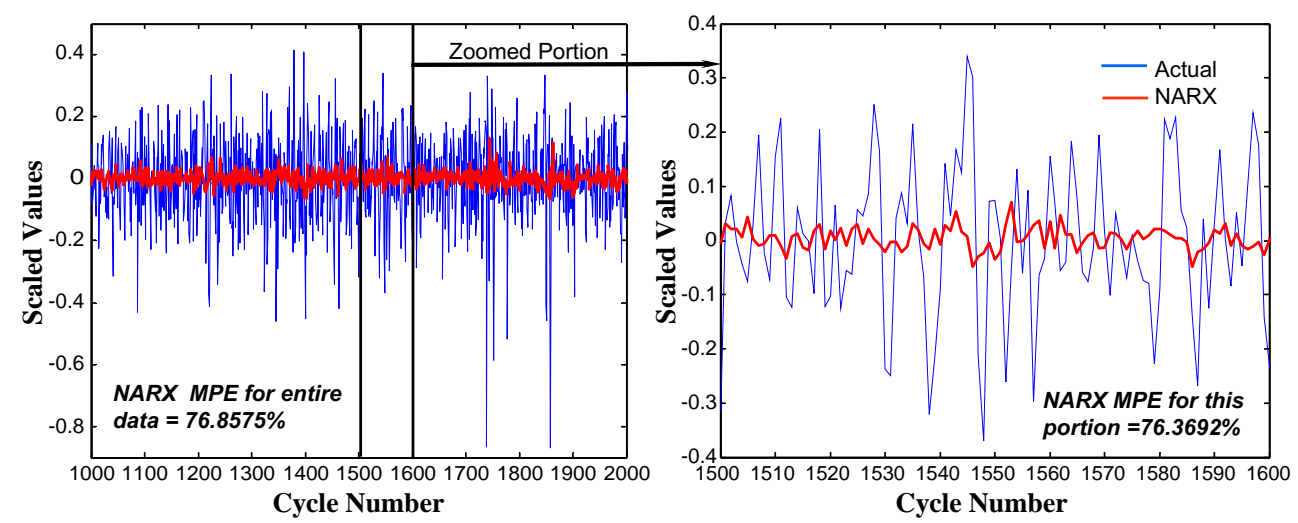

Fig. 17. NARX model of the high-frequency data for grindstone 1.

Table 2

Modelling results for high-frequency variation removal rate data

\begin{tabular}{lllllll}
\hline Data Set & $\operatorname{Var}\left(r_{k}\right)$ & $\operatorname{Var}\left(\Delta r_{k}\right)$ & $\begin{array}{l}\% \text { of } r_{k} \text { variation } \\
\text { represented by the H.F. } \\
\text { data }\end{array}$ & $\begin{array}{l}\text { \% of the H.F. data } \\
\text { explained by a linear } \\
\text { model }\end{array}$ & $\begin{array}{l}\text { \% of the H.F. data } \\
\text { explained by a NARX } \\
\text { model }\end{array}$ \\
\hline 1 & 0.0726 & 0.0187 & 25.7207 & 1.4731 & $\begin{array}{l}\text { Estimated } \\
\text { noise variance }\end{array}$ \\
2 & 0.1111 & 0.0152 & 13.6499 & 3.6762 & 1.8026 & 0.0183 \\
3 & 0.0964 & 0.0125 & 12.9675 & 3.9978 & 8.6986 & 0.0146 \\
4 & 0.0977 & 0.0116 & 11.8802 & 4.3767 & 4.5908 & 0.0114 \\
5 & 0.0984 & 0.0141 & 14.3105 & 5.7073 & 7.0959 & 0.0135 \\
\hline
\end{tabular}

control schemes are considered, starting with a simple open-loop controller and ending with a neural internal model controller. This incremental approach is adopted to give some insight into the underlying control problem and how it can be addressed. In particular, the openloop controller is included to show how the removal rate model can be used to implement an exact direct inverse model controller for process. This then provides the platform for developing more sophisticated model or inverse-model-based control.

Fig. 19 shows a block diagram of the disk grinding process highlighting the variables relevant to the thickness control problem. The unloading thickness, $t_{k}$, is governed by the current cycle time, $c_{k}$, the current pressure exerted onto the grindstones, $p_{k}$, and various unmeasured disturbances, $d_{k}$. The latter include coolant flow rate fluctuations, mechanical vibration, thermal effects and variation in the incoming disk thickness. The material removal rate, $r_{k}$ can be considered an internal process state which is a function of pressure and the unknown disturbances.

\subsection{Open-loop control}

A basic open-loop controller for the process can be implemented using $c_{k}$ as the manipulated input on the 

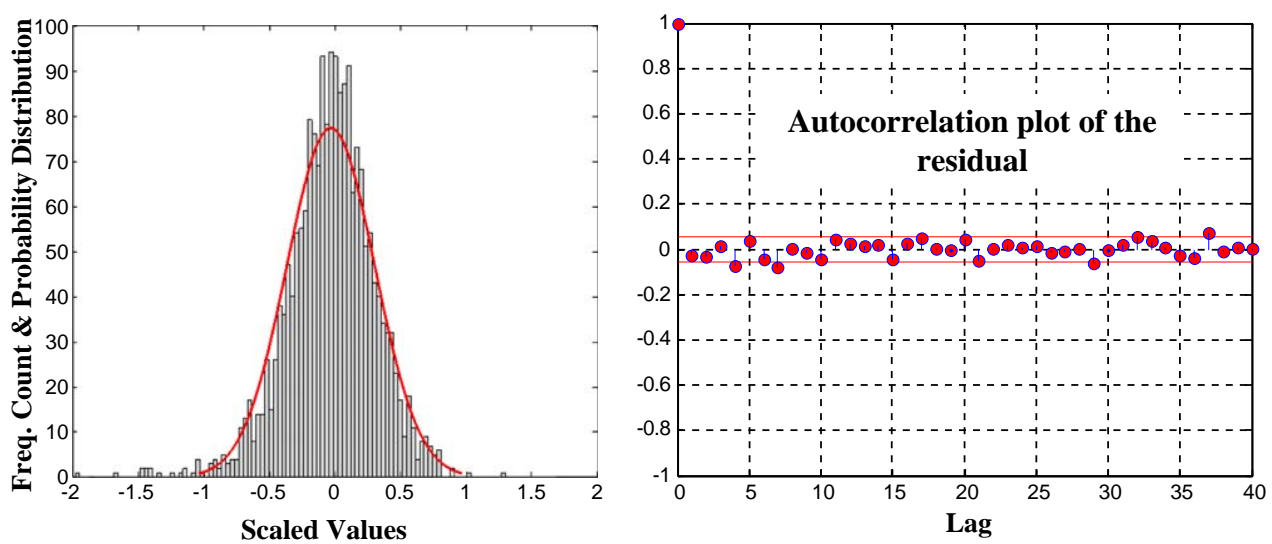

Fig. 18. Predicted residual normal distribution and autocorrelation plot for grindstone 1 model.

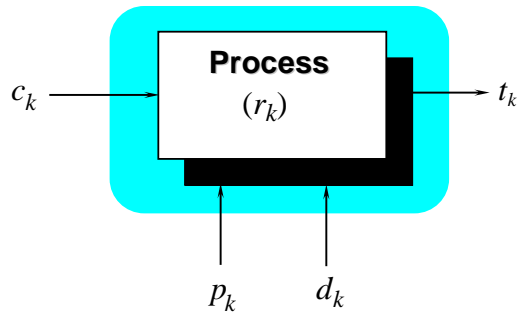

Fig. 19. The ring grinding process block diagram.

basis of an assumed constant removal rate, $\bar{r}_{k}$ (this could be chosen for example as the average removal rate over the past 100 cycles) (Fig. 19).

$c_{k}=\frac{t_{k}^{L}-t_{k}^{S P}}{\bar{r}_{k}}=\frac{\Delta t_{k}^{S P}}{\bar{r}_{k}}$.

Here $t_{k}^{L}$ is the measured loading thickness of the disks, $t_{k}^{S P}$ is the desired/set-point thickness and $\Delta t_{k}^{S P}$ is the thickness of material to be removed. In practice, however, the use of a constant removal rate value $\bar{r}_{k}$ would be a poor choice since grindstone performance deteriorates over time. To counter this, an aging factor can be incorporated into the $r_{k}$ estimate using for example an expression of the form

$r_{k}=\alpha r_{k-1}\left(\frac{p_{k}}{p_{k-1}}\right)$.

Here $\alpha$ is the aging rate, $|\alpha|<1$, and the ratio $p_{k} / p_{k-1}$ factors in the positive effect of ramping up the pressure following resurfacing (see Fig. 5).

Alternatively, the DNNM model identified previously can be used to provide an accurate estimate of $r_{k}$ at each iteration leading to the open-loop control scheme depicted in Fig. 20.

This in fact is a direct inverse model (IM) control implementation as may be deduced as follows. First, note that the DNNM removal rate model can also be used to generate a $c_{k}$-to- $t_{k}$ forward process model as shown in Fig. 21. Lee and Shin (2001) pointed out that an

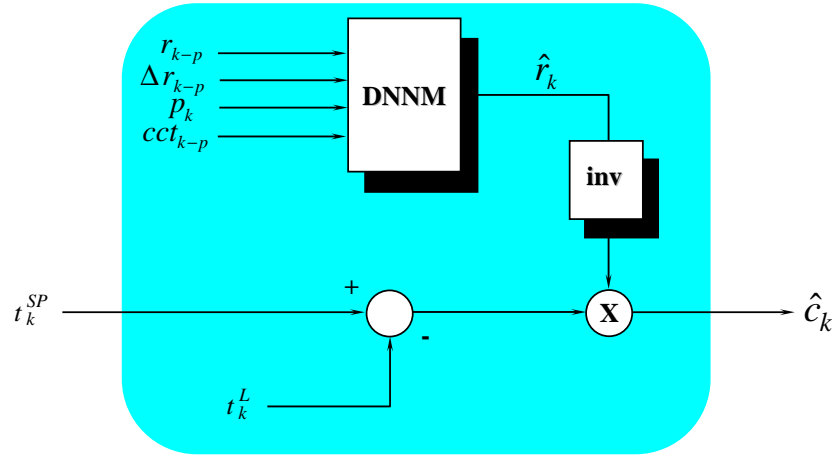

Fig. 20. Open-loop control using a ANN removal rate predictor.

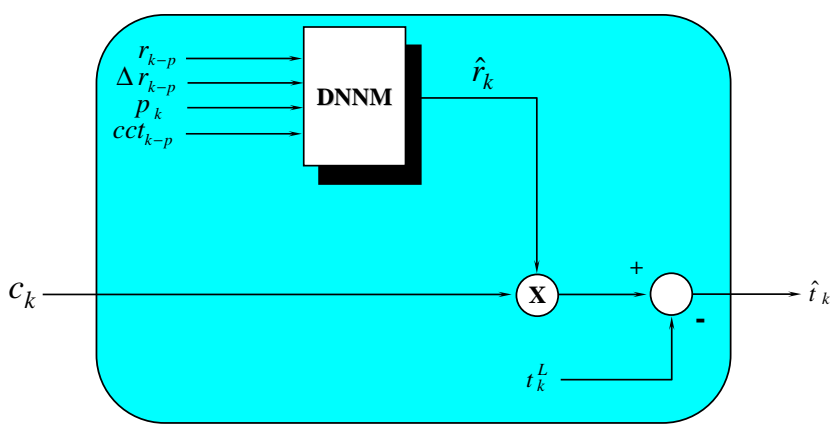

Fig. 21. Forward process model.

important feature of this particular formulation is that it allows the inverse plant model to be obtained without having to invert the ANN model, as is usually the case with a neural-control scheme (Hunt, Sbarbaro-Hofer, Zbikowski, \& Gawthrop, 1992). Thus, Fig. 20 represents an exact inverse of the forward process model and therefore a direct IM controller. The complete direct IM control scheme is therefore as shown in Fig. 22.

\subsection{Classical feedback control}

The controllers outlined in the previous section are open-loop controllers and therefore do not have a 


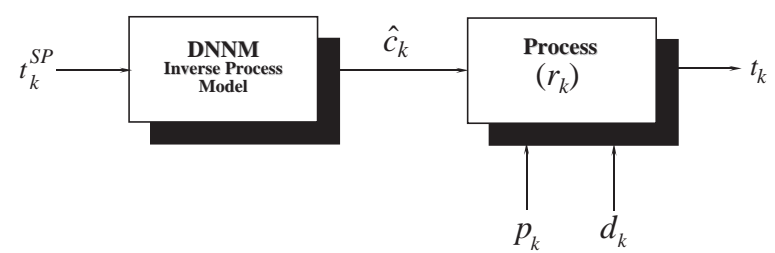

Fig. 22. Open-loop direct IM control using the NARX modelling method of the grinding process.

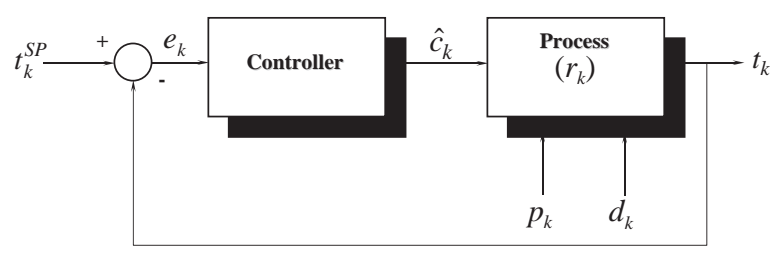

Fig. 23. Classical error feedback control using an integral action (IC).

mechanism to counter disturbances or model mismatch. This can be resolved by incorporating an error feedback loop. The control law here is typically of proportionalplus-integral (PI) controller form defined as

$c_{k}=c_{k-1}-K_{i} e_{k}-K_{p}\left(e_{k}-e_{k-1}\right)$,

where $K_{p}$ and $K_{i}$ are the proportional and integral gains, respectively (Fig. 23).

Classical error feedback control using an integral action (IC), the feedback error, $e_{k}$ is defined as

$e_{k}=t_{k}^{S P}-t_{k}$.

Note that the signs of the controller gains are negative to account for the negative gain of the process. From initial experiments it was found that the P term in the PI controller (Fig. 23 below) had little or no positive effect on thickness control and consequently the $\mathrm{P}$ term was omitted in the final implementation yielding an integral only controller (i.e. IC). According to Åström and Wittenmark (1997), special cases exist where an unknown constant disturbance acting on a plant can be controlled by just an integral action controller (IC). Incoming thickness variation is an example of such a disturbance.

\subsection{Feedforward direct neural inverse control with error feedback}

Both PC and feedback control with integral action (i.e. IC) have the same goal-removing the effect of lowfrequency and constant offsets, the former in the removal rate predictions, the latter in the tracking of the desired or set-point unloading thickness. These schemes can be combined with the direct IM control approach to provide the more advanced composite control scheme, shown in Fig. 24.

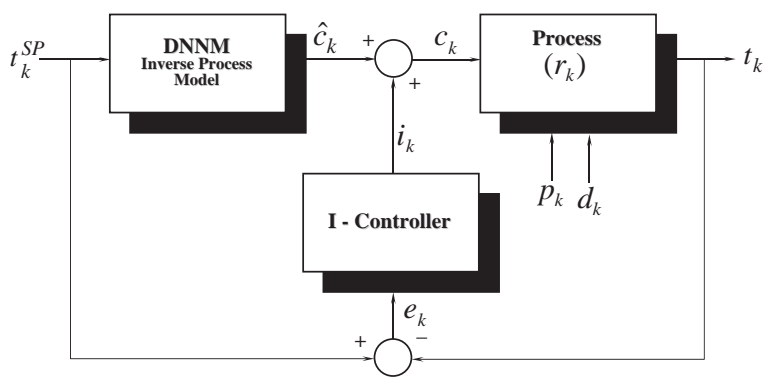

Fig. 24. The proposed neural direct IM control with error feedback compensation $(\mathrm{IM}+\mathrm{IC})$.

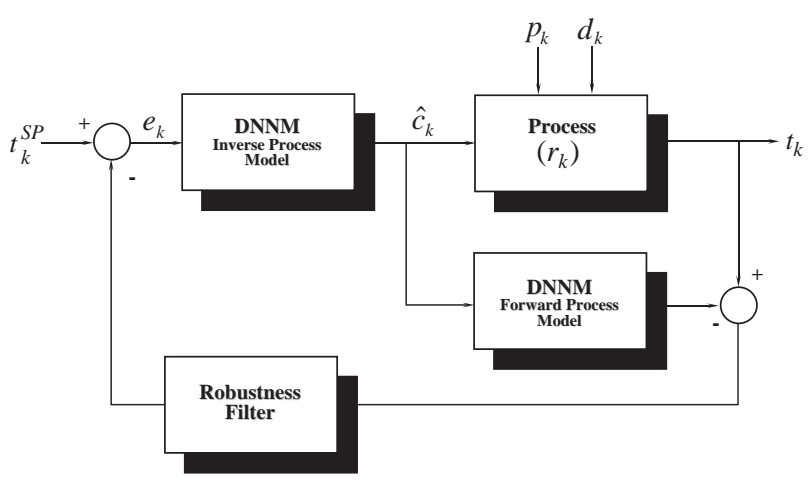

Fig. 25. The proposed internal model control (IMC) with a NARX Model scheme.

In this case the composite control is given by

$c_{k}=\hat{c}_{k}-i_{k}, \quad i_{k}=i_{k-1}-K_{i} e_{k}$,

where $\hat{c}_{k}$ is the estimate of the control action produced by the IM controller and $K_{i} e_{k}$ is the IC correction. The inclusion of the IC will be particularly effective at reducing the effects of disturbances such as incoming thickness variation.

\subsection{Neural internal model control}

The final control scheme considered is the wellknown, internal model control (IMC) scheme. This has been extensively studied for linear systems control, and has proven to be robust against disturbances and model mismatch (Zafiriou \& Morari, 1991). Nonlinear implementations of this scheme have also proven effective, e.g. Psichogios and Ungar (1991), Hunt and Sbarbaro (1991), Hunt et al. (1992) and Kalkkuhl and Hunt (1996), and Lightbody and Irwin (1997).

This method has similarities to the composite openloop direct IM control scheme in Section 5.1. The main difference is that the control action of the IM controller is an input to a neural network model as well as to the actual plant. The difference between the actual unloading thickness, $t_{k}$, and the predicted unloading thickness, $\hat{t}_{k}$, is then fed back to the controller, as shown in Fig. 25. A set-point tracking/model mismatch signal is thus 
available to the controller. Provided the controller is an exact inverse of the model, which is guaranteed with the adopted model structure, the system has zero steadystate error. Lightbody and Irwin (1997) provide some theoretical results on neural IMC.

The use of the robustness filter offers disturbance response performance. Here, a first-order exponential filter given by

$F(z)=\frac{\left(1-\mathrm{e}^{-1 / \tau}\right) z^{-1}}{1-\mathrm{e}^{-1 / \tau} z^{-1}}$

was adopted. The filter time constant, $\tau$, was chosen to obtain optimum closed-loop performance as describe in Section 6 .

\section{Results}

The experimental results were obtained using actual historical data gathered over 5 grindstone lifetimes to gauge the performance of the various process optimisation schemes presented here. Several performance criteria were chosen. These included the mean and standard deviation of the unloading thickness error, the number of rejects (i.e. disks exceeding either the upper or lower unloading thickness specifications) and the process capability index, $C_{p k}$, which is defined as follows (Degarmo, Black, \& Kosher, 1990; Lee \& Shin, 2002);

$C_{p k}=\frac{\min \{|U C L-\bar{X}|,|L C L-\bar{X}|\}}{3 \sigma}$.

In Eq. (6.1), UCL and LCL are the upper and lower control limits of the unloading thickness, while $\bar{X}$ and $\sigma$ are the mean and the standard deviation of the unloading thickness, respectively. If $C_{p k} \geqslant 1$, then at least $99.7 \%$ of all products of the process will be within specification limits while $C_{p k}<1$, some non-conforming disks are being produced. Table 3 summarises the performance of the different control schemes described in Section 5, on each of the 5 grindstone data sets. The results presented are the combined performance values, which have been averaged and normalised over all the data sets.

Results for Seagate's proprietary control scheme and the static ANN direct inverse control method proposed by Lee and Shin (2001) are also included for comparison. The proprietary control scheme is based on an IC whose gain varies as a function of the removal rate. However, due to confidentiality issues, the details of the algorithm cannot be disclosed here. The parameters of the control schemes proposed were optimised using the first 600 data points of each grindstone (approximately $25 \%$ of the data) and subsequently evaluated on the remainder of the data. For the IC controller, $K_{i}$ was set between $0.0004>K_{i}>0.0005$ for each of the grindstones. Meanwhile the time constant, $\tau$, for the robustness filter, $F(z)$, of the IMC scheme was identified as $0.85>\tau>0.88$.

While Lee and Shin (2001) reported good results with their control scheme, it is clear that it does not provide satisfactory performance for the ring grinding machine investigated here. This may be because their process did not have significant dynamic variations, or it may be that the online adaptation procedure they have employed to obtain an adaptive direct inverse controller compensated for the unmodelled dynamics of the process.

Figs. 26-28 compare the unloading thickness distribution of the proprietary control scheme with those obtained using IC, Lee and Shin's controller and IMC. The thickness distribution obtained with the NARX model control scheme clearly shows that tighter thickness control is achieved, that is, there is less variation in the unloading thickness. Fig. 29 provides a graphical comparison of the $C_{p k}$ and the rejects reduction ratio, $\phi_{x}$, for each controller. The latter is defined as

$\phi_{x}=\frac{\text { Rejects with proprietry control }}{\text { Rejects with proposed controller } x}$.

This again reflects the superiority of the DNNM-based control schemes in reducing the number of defects compared to the alternatives.

Clearly from the results above, it can be seen that the DNNM model-based control are significantly better

Table 3

Performance summary of all the optimisation techniques $(\mathrm{IC}=$ integral control, $\mathrm{IM}=$ direct inverse model control, $\mathrm{IM}+\mathrm{PC}=$ direct inverse model with predict correct control, IM + IC $=$ direct inverse model with integral control, $\mathrm{IM}+\mathrm{PC}+\mathrm{IC}=$ direct inverse model with predict correct and integral control, IMC $=$ internal model control)

\begin{tabular}{lcccc}
\hline Control scheme & Mean of the thickness deviation & Std. of the thickness deviation & Ratio of improvement on rejects & Norm. $C_{p k}$ \\
\hline Proprietary & 17.7318 & 16.0271 & - & 0.31 \\
Lee and Shin & 4.0046 & 22.7474 & 0.76 & 0.66 \\
IC & 0.0684 & 20.9696 & 2.99 & 0.46 \\
IM & 2.7073 & 12.6789 & 2.95 & 0.46 \\
IM + PC & 2.6570 & 12.6803 & 2.62 & 0.81 \\
IM + IC & 1.2927 & 13.0698 & 2.07 & 0.76 \\
IM + PC + IC & 0.3901 & 13.1431 & 2.750 & 0.74 \\
IMC & 0.1226 & 12.7508 & & 0.76 \\
\hline
\end{tabular}




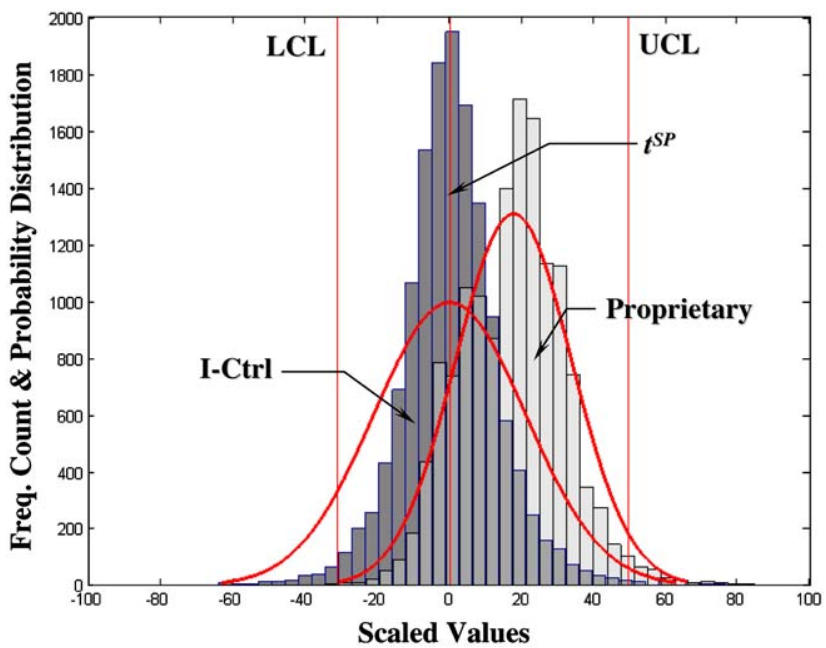

Fig. 26. Unloading thickness distribution plot of the I-Control (dark grey) compared to the proprietary (light grey).

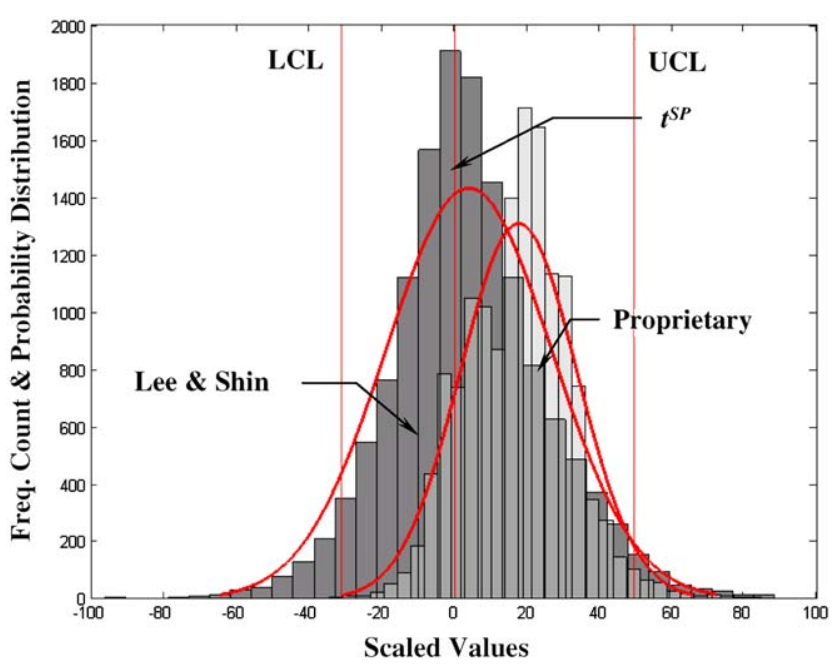

Fig. 27. Unloading thickness distribution plots of Lee and Shin's method (dark grey) compared to the proprietary (light grey).

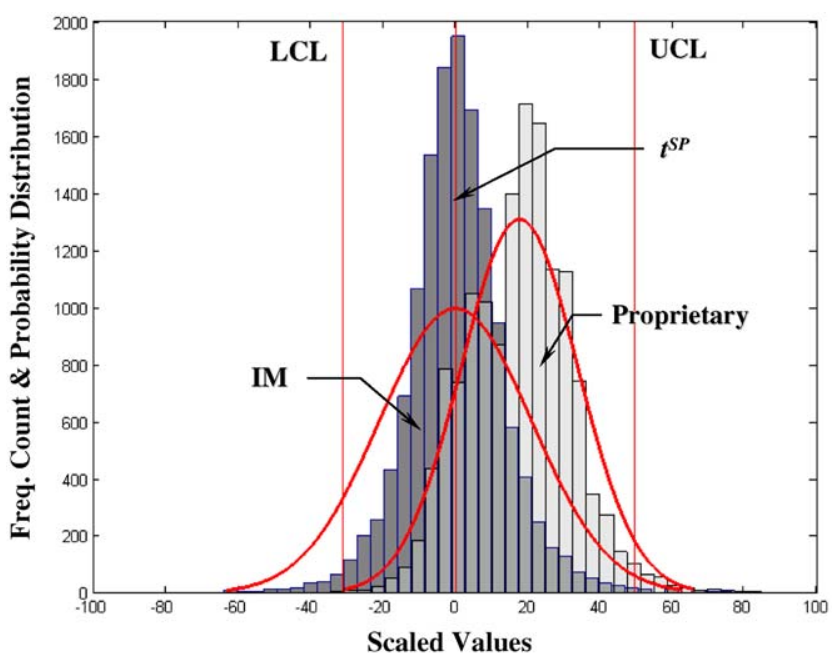

Fig. 28. Unloading thickness distribution plot of the IM method compared to the proprietary scheme. than the other schemes studied. However, in practice, there was little significant difference in performance between the different implementations. This is a reflection of the quality of the process model obtained, in that the model has sufficiently captured all the dynamic characteristics of the ring grinding process, leaving nothing for the controllers to do.

However, should a disturbance occur in the process, control schemes with error feedback can be expected to out-perform the open-loop controllers. One possible source of disturbance is the loading thickness of the aluminium substrate disks. Currently this is measured at each grind cycle but the company is considering omitting this measuring phase to speed up the overall productivity (this task normally takes about $0.1 \%$ of the total production cycle) and to rely on the suppliers providing the disks within pre-specified loading thickness tolerances.

To gauge the effectiveness of the various controllers for this scenario, their performance has been evaluated on historical data from grindstone 1. A disturbance was simulated by introducing an undetected $3 \sigma$ deviation change in the disk loading thickness, $\frac{1}{3}$ of the way through the grindstone life. Unloading thickness timehistories obtained with the IMC and IM controllers in response to this disturbance are provided in Fig. 30. Table 4 provides a summary of the performance of all control schemes considered for this disturbance. The equivalent results without the disturbance are also included for comparison. As expected, the control schemes incorporating error feedback effectively reject the disturbance with IMC providing the best overall performance.

\section{Conclusions and future work}

Neural network-based modelling and control have been used to optimise an industrial grinding process used in machining of aluminium disks, a key component of computer hardisk drives. Linear and nonlinear dynamic models of the grindstone removal rate have been studied and the key findings were as follows:

1. Linear models were unsatisfactory, indicating that the underlying removal rate dynamics were nonlinear.

2. Including local dynamic variations improved model accuracy, while modelling the high-frequency variations separately reduced the model order.

3. Nonlinear dynamic models, in the form of generalised multilayer perceptron NARX-based structures, provided an ideal framework for building on a priori knowledge in the form of linear modelling experience and produced good results.

4. While most of the high-frequency variation in removal rate could not be characterised by the 


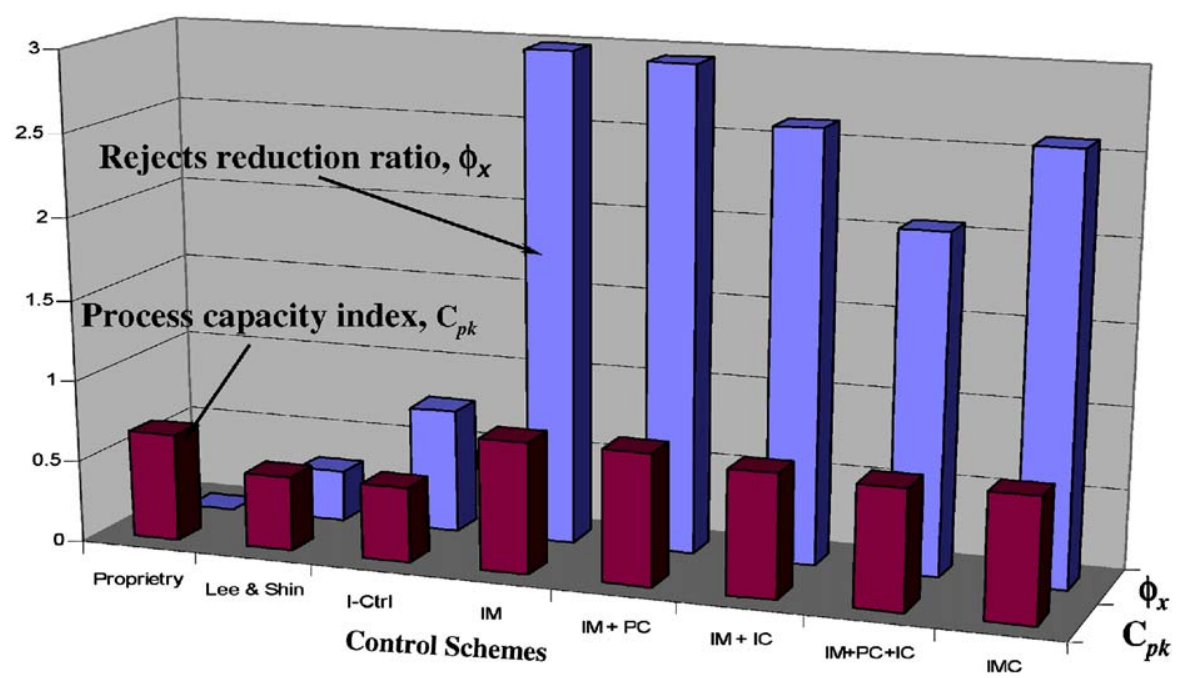

Fig. 29. Process capacity index, $C_{p k}$ and rejects reduction ratio, $\phi_{x}$, comparison for the different control schemes employed.

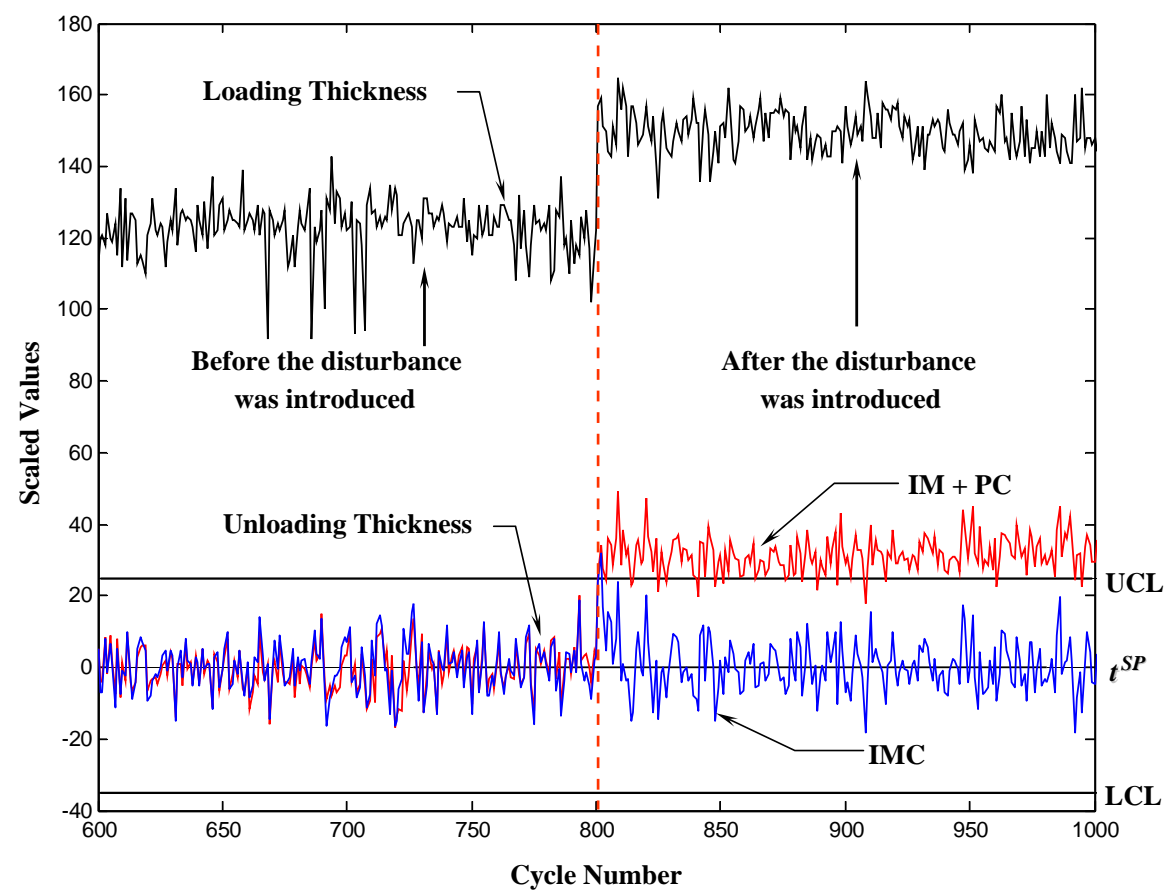

Fig. 30. Step change disturbance performance comparison of the open-loop inverse controller with predict correct (IM + PC) and the internal model controller (IMC).

Table 4

Disturbance rejection performance comparison

\begin{tabular}{|c|c|c|c|c|c|c|}
\hline \multirow[t]{2}{*}{ Control scheme } & \multicolumn{3}{|l|}{ Without disturbance } & \multicolumn{3}{|l|}{ With disturbance } \\
\hline & $\begin{array}{l}\text { Normalised mean of } \\
\text { the thickness } \\
\text { deviation }\end{array}$ & $\begin{array}{l}\text { Normalised std. of the } \\
\text { thickness deviation }\end{array}$ & $\begin{array}{l}\text { Normalised } \\
C_{p k}\end{array}$ & $\begin{array}{l}\text { Normalised mean of } \\
\text { the thickness } \\
\text { deviation }\end{array}$ & $\begin{array}{l}\text { Normalised std. of the } \\
\text { thickness deviation }\end{array}$ & $\begin{array}{l}\text { Normalised } \\
C_{p k}\end{array}$ \\
\hline Lee and Shin & 1.2495 & 8.5909 & 0.9215 & 22.8875 & 17.7152 & 0.0397 \\
\hline IC & 0.0507 & 8.8028 & 0.9447 & 0.1037 & 9.5463 & 0.8693 \\
\hline IM & 1.3283 & 6.3109 & 1.2503 & 22.9663 & 16.4221 & 0.0413 \\
\hline $\mathrm{IM}+\mathrm{PC}$ & 1.2353 & 6.2836 & 1.2607 & 22.8732 & 16.3645 & 0.0433 \\
\hline $\mathrm{IM}+\mathrm{IC}$ & 0.0499 & 6.3488 & 1.3100 & 0.1651 & 7.7799 & 1.0641 \\
\hline $\mathrm{IM}+\mathrm{PC}+\mathrm{IC}$ & 0.0496 & 6.3374 & 1.3123 & 0.1649 & 7.7670 & 1.0658 \\
\hline IMC & 0.0027 & 6.8595 & 1.2147 & 0.0496 & 8.2328 & 1.0102 \\
\hline
\end{tabular}


measured variables, analysis indicated that this component could be regarded as Gaussian white noise.

Various model-based control strategies were investigated and compared with the existing proprietary control scheme. These included direct inverse control, direct inverse control with error feedback and internal model control (IMC). Results showed that:

1. Much tighter control of the variation in the unloading thickness was achieved using such controllers, with the number of rejects reduced by a factor of two or more.

2. There was little significant difference between the performances of the neural open-loop and errorfeedback control schemes, indicating the absence of significant disturbances in the current grinding process.

3. Using a simulated step change in disk loading thickness it was confirmed that the error-feedback controllers were superior at rejecting disturbances. The dynamic neural network-based IMC scheme provided the best overall performance.

On the whole the results of this investigation suggest that the ring grinding process can be effectively optimised by employing dynamic neural network model-based control. The authors are currently looking into implementing these methods on other grinding processes within the aluminium subtrate disk industry.

\section{Acknowledgement}

The first author wishes to acknowledge the technical and financial support of Seagate Technology Media (Ireland) Ltd. and Queen's University Belfast.

\section{References}

Åström, K. J., \& Wittenmark, B. (1997). Computer-controlled systems: Theory and designs (3rd ed.). Prentice-Hall Information and System Sciences Series, 1997.

Brinkmeier, E., Tšnshoff, H. K., Czenkusch, C., \& Heinzel, C. (1998). Modelling and optimization of grinding processes. Journal of Intelligent Manufacturing, 9, 303-314.

Chen, Y. T., \& Kumara, S. R. T. (1998). Fuzzy logic and neural networks for design of process parameters: a grinding process application. International Journal of Production Research, 36(2), 395-415.

Chryssolouris, G., Guillot, M., \& Domroese, M. (1988). A decision making approach to machining control. Journal of Engineering for Industry, ASME, 110, 397-398.

Cybenko, G. (1989). Approximation by superpositions of a sigmoidal function. Mathematics of Control, Signals, and Systems, 2, 303-314.

Degarmo, E. P., Black, J. T., \& Kosher, R. A. (1990). Materials and processes in manufacturing (7th ed.). Reading, NJ: Prentice-Hall.
Dote, Y., \& Ovaska, S. P. (2001). Industrial applications of soft computing: A review. Proceedings of the IEEE, 89(9), 1243-1265.

Heikkonen, J., \& Lampinen, J. (1999). Building industrial applications with neural networks. Proceedings of the European symposium on intelligent techniques. Orthodox Academy of Crete, Chania, Greece, June 3-4, 1999.

Hornik, K. (1989). Multilayer feedforward networks are universal approximators. Neural Networks, 2, 359-366.

Hunt, K. J., Irwin, G. W., \& Warwick, K. (Eds.) (1995). Neural network engineering in dynamical control systems. Advances in industrial control series. Berlin: Springer.

Hunt, K. J., \& Sbarbaro, D. (1991). Neural networks for nonlinear internal model control. IEE Proceedings-Part D: Control Theory and Applications, 138(5), 431-438.

Hunt, K. J., Sbarbaro-Hofer, D., Zbikowski, R., \& Gawthrop, P. J. (1992). Neural networks for control systems - a survey. Automatica, 28, 1083-1112.

Hussain, M. A. (1999). Review of the applications of neural networks in chemical process control - simulation and online implementation. Artificial Intelligence in Engineering, 13(1), 55-68.

Irwin, G. W., O'Reilly, P., Lightbody, G., Brown, M., \& Swidenbank, E. (1995a). Electrical power and chemical process applications. In Irwin, G. W., Warwick, K., \& Hunt, K. J. (Eds.), Neural network applications in control. IEE control engineering series 53. London, UK: The Institution of Electrical Engineers.

Irwin, G. W., Warwick, K., \& Hunt, K. J. (Eds.) (1995b). Neural network applications in control. IEE control engineering series 53. London, UK: The Institution of Electrical Engineers.

Kalkkuhl, J. C., \& Hunt, K. J. (1996). Discrete-time neural model structures for continuous nonlinear systems: fundamental properties and control aspects. In Zbikowski, R., \& Hunt, K. J. (Eds.), Neural adaptive control, Vol. 1 (pp. 3-40). Singapore: World Scientific.

Lee, C. W., \& Shin, Y. C. (2001). Intelligent modelling and control of computer hard disk grinding processes. Proceedings of the third international conference on intelligent processing and manufacturing of materials (pp. 829-838). Vancouver, Canada, July 29th-August 2nd, 2001.

Lennox, B., Montague, G. A., Frith, A. M., Gent, C., \& Bevan, V. (2001). Industrial application of neural networks - an investigation. Journal of Process Control, 11(5), 497-507.

Liao, W. T., \& Chen, L. J. (1994). A neural network approach for grinding processes: modelling and optimization. International Journal of Machine, Tools and Manufacturing, 34(7), 919-937.

Lightbody, G. (1993). Identification and control using neural networks. Ph.D. dissertation. The Intelligent Systems and Control Group, The Queen's University of Belfast, Northern Ireland, UK.

Lightbody, G., \& Irwin, G. W. (1997). Nonlinear control structures based on embedded neural system models. IEEE Transactions on Neural Networks, 8(3), 553-567.

Masters, T. (1995). Neural, novel \& hybrid algorithms for time series prediction (p. 103). New York: Wiley.

McLoone, S. F., \& Irwin, G. W. (1997). Fast parallel off-line training of multilayer perceptrons. IEEE Transactions on Neural Networks, $8(3), 646-653$.

Merchant, M. E. (1998). An interpretive look at 20th century research on modelling of machining. Proceedings of the first CIRP international workshop on modelling of machining operations (pp. 27-31). Atlanta, GA, USA, May 19, 1998.

Psichogios, D. C., \& Ungar, L. H. (1991). Direct and indirect model based control using artificial networks. Industrial and Engineering Chemistry Research, 30(12), 2564-2573.

Rovlak, J. A., \& Corlis, R. (1991). Dynamic matrix based control of fossil power plants. IEEE Transactions on Energy Conversion, 6(2), 320-326. 
Seagate Technology: Company Facts Sheet (2002). http://www.seagate.com/newsinfo/about/profile/D1al.html.

Shin, Y. C., Chen, Y. T., \& Kumara, S. (1992). Framework of an intelligent grinding process advisor. Journal of Intelligent Маnufacturing, 3, 135-148.

Shin, Y. C., \& Vishnupad, P. (1996). Neuro-fuzzy control of complex manufacturing processes. International Journal of Production Research, 34(12), 3291-3309.

Sjöberg, J. (1997). On estimation of nonlinear black-box models: how to obtain a good initialization. Report CTH-TE-60 (Proceeding of IEEE workshop in neural networks for signal processing. Amelia Island Plantation, Florida, September 24-26).

Udo, G. J. (1992). Neural networks applications in manufacturing processes. Computers and Industrial Engineering, 23(1-4), 97-100.
Warwick, K., Irwin, G. W., \& Hunt, K. J. (Eds.) (1992). Neural networks for control and systems. IEE control engineering series 46 . London, UK: Peter Peregrinus.

Willis, M. J., Di Massimo, C. D., Montague, G. A., Tham, M. T., \& Morris, A. J. (1991). Artificial neural networks in process engineering. IEE Proceedings-Part D: Control Theory and Applications, 138(3), 256-266.

Zafiriou, E., \& Morari, M. (1991). Internal model control: Robust digital controller synthesis for multivariable open-loop stable or unstable processes. International Journal of control, 54(3), 665-704.

Zhang, H. C., \& Huang, S. H. (1995). Applications of neural networks in manufacturing: a state of the art review. International Journal of Production Research, 33, 705-728. 